\title{
Light-related Electrochemistry in the 21st Century
}

\section{Kazuhito HASHIMOTo}

During the $20^{\text {th }}$ century, humankind was able to develop an extremely comfortable lifestyle thanks to developments

in science and technology. The price to pay, however, was the rampant and massive consumption of precious fossil

fuels that were formed and preserved during the Earth's 4.6 billion-year history. Rapid consumption of these fossil

fuels in the late $20^{\text {th }}$ century has resulted in an energy crisis and probably global warming, problems which are intri-

cately tied to the very survival of the human species.

In light of this situation, the science and technology of the $21^{\text {st }}$ century must follow a different track than the sci-

ence and technology of the previous century. Rather than a model based on consumption, we must create a model based on sustainability. In other words, energy must not be regarded as merely a resource that we "consume," but a commodity that we "create and use."

It is clear that electrochemistry plays an extremely important role in both energy production and its transportation stages. In particular, the electrochemistry concerned with light may potentially provide completely novel methodology towards the development of new energy systems. Fusion of light-related electrochemistry with newly developed technologies in nanotechnology and biotechnology may allow these systems to be realized. In the $21^{\text {st }}$ century, we electrochemists should wrestle with these difficult but challenging issues.

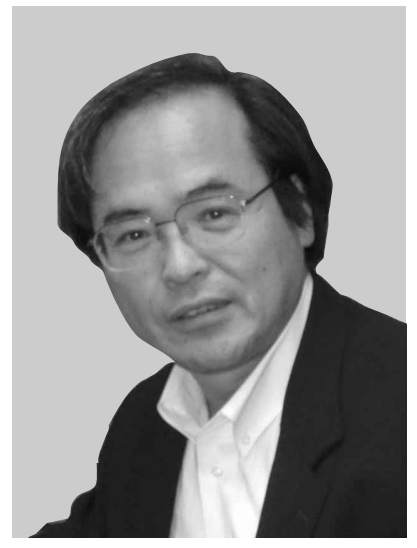

Kazuhito Hashimoto Professor, The University of Tokyo 